\title{
Managen im Spital - Spitäler investieren in Führung
}

\author{
C. Schmitz
}

Führung ist matchentscheidend. Das gilt auch für Spitäler. Sowohl das Kantonsspital Basel als auch das Inselspital Bern haben sich darum in Kooperation mit dem College-M entschlossen, eigene Programme für eine kohärente, institutionsspezifische und berufsgruppenübergreifende Führungsausbildung zu etablieren.

Korrespondenz:

Christof Schmitz

College-M

Freiburgstrasse 41

CH-3010 Bern

Internet: www.college-m.ch
Führungsausbildung war in Spitälern bislang weitgehend individueller Initiative oder berufsgruppenspezifischen Ausprägungen überlassen. Nach wie vor ist es eher die Ausnahme, dass sich ein Spital entschliesst, eine auf die eigene spezifische Situation passende Führungsausbildung aufzusetzen. Das Fehlen solcher Programme drückt sich im Mangel an kohärenten Führungsverständnissen aus. Dieses Manko fällt insbesondere dann ins Gewicht, wenn anspruchsvolle Veränderungen und Entwicklungen in die Wege geleitet und umgesetzt werden sollen. Viel Energie und Einsatz verpufft heute nutzlos, und dies ist für die Beteiligten frustrierend, weil die entsprechenden gemeinsamen Verständnisse und Methoden nicht ausreichend zur Verfügung stehen.

Führungsausbildungen sind nicht Bestandteil medizinischer Ausbildungen, abgesehen von der Tradition der Kaderschulen in der Pflege. Allein von daher besteht Nachholbedarf, befinden sich doch auch Ober-, Leitende und Chefärzte offensichtlich in anspruchsvollen Führungspositionen. Sie sehen sich neben ihren fachlichen nisieren, Menschen fördern, Leistungen messen und bewerten usw. konfrontiert. Diese sind genuine Managementaufgaben. Zusehends beginnen Spitäler, von ihrem Kader Kompetenz auch in diesen Belangen einzufordern. «Managementund Führungskompetenzen werden auch in der Ärzteschaft immer wichtiger und sind eine Ergänzung zum Arztsein. Management und Arztsein sind aber auch spannungsreiche Pole.» (Prof. Dr. med. A. Tobler, Ärztlicher Direktor, Inselspital Bern)

\section{Kohärent}

Die Qualität von Führung ist essentiell für den Erfolg einer Organisation. Offensichtlich gibt es nicht das eine, verbindliche Rezept, das Führungserfolg garantieren würde. Führung ist eine anAufgaben mit solchen wie Ziele definieren, Orga- spruchsvolle und komplexe Aufgabe, die vielfältige Wege und Mittel ergreifen muss. So vielfältig sind denn auch die Führungsansätze, die heute zu finden sind. Natürlich ist es auch ein Gewinn, wenn jemand sich individuell mit Führung beschäftigt. Doch ist dieser Gewinn für eine Institution um so grösser, je kohärenter die Verständnisse sind, auf die sich Führungsarbeit insgesamt beziehen kann. Erst dann kann von der Ausbildung einer gemeinsam geteilten (Führungs-)Kultur gesprochen werden, die es braucht, um heutige Veränderungsprozesse erfolgreich meistern zu können. Und erst dann kann geklärt werden, was von Führung tatsächlich erwartet werden kann.

\section{Institutionsspezifisch}

Sowohl das Inselspital (seit 2003) als auch das Kantonsspital Basel (seit 2002) haben sich entschlossen, institutionsspezifische Führungsprogramme zu lancieren. Die Programme sind auf die konkrete Situation und Anforderungen des jeweiligen Spitals bezogen. Vertreter der Spitalleitung sowie andere Referenten aus den Häusern treten in den Modulen auf, stellen spitalrelevante Inhalte vor und diskutieren mit den Teilnehmern konkrete Fragen und Anmerkungen. Dabei wird nicht nur institutionsspezifisches Wissen an die Teilnehmenden vermittelt, sondern auch die Referenten gewinnen Eindrücke und erhalten Rückmeldungen.

\section{Berufsübergreifend}

Wie kaum ein anderes ist das Gesundheitssystem durch seine Berufsgruppen tief geprägt. Die Dominanz der jeweiligen professionellen Orientierungen ist nicht $\mathrm{zu}$ übersehen und blockiert nicht selten die Lösung wichtiger Probleme. So bedeutet beispielsweise Führung bei Ärzten regelmässig etwas anderes als in der Pflege oder der Verwaltung. Im berufsübergreifenden Ansatz wird sowohl deutlich, wie ähnlich relevante führungsbezogene Fragestellungen sein können, als auch, welche Unterschiede in den jeweiligen Subkulturen bestehen. Auch wird erfahrbar, wel- 
che Bereicherung die Nutzung der Vielfalt der vorhandenen Perspektiven bedeuten kann. Die kommenden grossen Herausforderungen in Gesundheitsinstitutionen werden in weiten Teilen interdisziplinär zu leisten sein. Auch darum hat die berufsübergreifende Besetzung grosse Bedeutung.

Die beiden Universitätsspitäler haben sich entschlossen, zu diesen Fragen eine Kooperation mit dem College-M in Bern einzugehen. «Das College-M ist ein innovativer Ausbildungspartner, der uns professionell und zielgruppenorientiert bei der Vermittlung von Managementund Führungskompetenzen für Kaderangestellte unterstützt.» (A. Bernhard, Leiterin Personalund Organisationsentwicklung, Kantonsspital Basel)
Das 1999 gegründete College für Management im Gesundheitswesen, College-M, ist eine unabhängige und gesamtschweizerische Stiftung der Verbände der Gesundheitsberufe (FMH, Labmed, SBK, SPV, SVBG) mit Sitz in Bern. Wir verstehen uns als Think-Tank und als Bildungs- und Forschungsunternehmen für Personen und Organisationen im Gesundheitswesen. Wir verfolgen einen integrativen Ansatz sowie eine eigene Lernkultur, orientieren uns an Interdisziplinarität sowie dem «State of the art» und legen Wert auf nationale und internationale Vernetzung.

Unsere Kernbereiche sind:

- Managementweiterbildungen für Führungspersonen aus Gesundheitsorganisationen;

- Forschung und Entwicklung zu neuartigen Integrations- und Steuerungsmodellen der Gesundheitsversorgung;

- Unterstützung von Personen und Institutionen in konkreten Entwicklungsprojekten. 